\title{
Européanisation et gouvernement démocratique
}

\section{Fritz W. Scharpf}

\section{Citer ce document / Cite this document :}

Scharpf Fritz W. Européanisation et gouvernement démocratique. In: Politiques et management public, vol. $15, \mathrm{n}^{\circ} 1,1997$. L'européanisation des politiques publiques : politiques communautaires et management public. Actes du huitième colloque international organisé avec le concours de la Commission Européenne et du Ministère de l'Education Nationale, de l'Enseignement Supérieur et de la Recherche (DISTNB) Paris - 20-21 juin 1996 - Tome I - Acteurs et processus des politiques européennes. pp. 11-20;

doi : https://doi.org/10.3406/pomap.1997.2130

https://www.persee.fr/doc/pomap_0758-1726_1997_num_15_1_2130

Fichier pdf généré le 22/04/2018 


\section{EUROPÉANISATION ET GOUVERNEMENT DÉMOCRATIQUE}

\section{Fritz W. SCHARPF *}

Le déficit démocratique existe

- Max-Plank Institut für Gesellschaft Forschung, Cologne.

Revue POLITIQUES ET MANAGEMENT PUBLIC, Volume 15, nº 1, mars 1997.

(C) Institut de Management Public - 1997.

La préoccupation du public sur le 'déficit démocratique' européen est d'origine récente. Elle a attiré l'attention dans les controverses qui ont conduit aux référendums sur Maastricht au Danemark et en France, et elle s'est ensuite élevée au statut d'une objection constitutionnelle fondamentale de la cour constitutionnelle fédérale en Allemagne dans son jugement sur Maastricht. Du point de vue de la théorie démocratique, cependant, ce débat est insuffisant pour autant qu'il se centre sur le déficit démocratique de l'Union européenne, plutôt que sur le déficit démocratique en Europe. On suppose, en d'autres termes, que la démocratie continue à prospérer au niveau national et que le déficit disparaîtrait si seulement la souveraineté nationale pouvait être défendue contre l'extension ultérieure des compétences européennes. A mon avis, ce point de vue souffre d'une conceptualisation incomplète de la démocratie.

La démocratie vise à une auto-détermination collective. Elle doit ainsi être comprise comme un concept bi-dimensionnel, en ce qui concerne à la fois les inputs et les outputs du système politique. Du côté des inputs, l'auto-détermination requiert que les choix politiques devraient être dérivés, directement ou indirectement, des préférences authentiques des citoyens et que, pour cette raison, les gouvemements doivent être tenus comme redevables aux gouvernés. Du côté des outputs, cependant, l'autodétermination implique un contrôle efficace du destin des sociétés. La démocratie serait un rituel vide si les choix politiques des gouvemements n'étaient pas capables d'atteindre un haut niveau d'efficacité dans la réalisation des objectifs, tout en évitant les dangers concemant collectivement les citoyens. Ainsi, l'authenticité orientée vers les inputs et l'efficacité orientée vers les outputs sont des éléments également essentiels de l'auto-détermination démocratique. 
Le débat actuel est insuffisant parce qu'il se centre exclusivement sur la faiblesse des structures dinput au niveau de l'Union européenne. C'est assez évident. Le Parlement européen est élu directement, mais son influence sur la législation européenne est très limitée. Plus important encore, même si les compétences du Parlement européen étaient élargies, cela n'aiderait pas beaucoup, parce que d'autres conditions, essentielles à l'authenticité démocratique, manquent également : il n'y a pas encore de partis politiques européens, pas de dirigeants politiques européens et aucun média de communication politique à l'échelle de l'Europe. En conséquence, nous n'avons aucune controverse, ni aucun débat au niveau européen sur les problèmes politiques et sur les choix de politiques, et nous n'avons aucune concurrence au niveau européen pour les fonctions de gouvemement. En l'absence de toutes ces conditions structurelles préalables au processus démocratique, le principe de majorité en luimême ne peut pas porter seul la légitimité démocratique. Ainsi, même si le Parlement européen avait tous les pouvoirs habituels des parlements nationaux, son vote ne pourrait pas légitimer des choix de politiques qui vont à l'encontre des intérêts ou des préférences profondément ancrées des citoyens dans certains des Etats membres. Et ce serait bien sûr encore plus vrai pour les votes de majorité au Conseil de Ministres.

Cette limitation est reconnue par des théories récentes mettant l'accent sur les sources non-majontaires de la légitimité des choix de politiques européens. Au lieu de responsabilité démocratique, c'est l'autorité de la loi et, plus généralement, l'autorité de l'expertise technique qui est supposée être importante. L'Union européenne, de ce point de vue, devrait ainsi être comprise non pas comme une forme imparfaitement démocratique de gouvernement, mais comme un "Etat régulateur" exerçant des pouvoirs similaires à ceux exerçés par les agences régulatrices indépendantes aux Elats-Unis, par des cours constitutionnelles, ou par des banques centrales indépendantes. Cependant, comme l'a remarqué Giandomenico Majone, l'auteur qui a le plus contribué à l'explication de ce phénomène, cette légitimité non-démocratique constitue un objectif hors d'atteinte. Cela présuppose un consensus normatif sur la validité de certaines normes, ou la désirabilité de certains résultats, et cela présuppose aussi l'existence de normes professionnelles par lesquelles il est possible de juger l'interprétation et l'application de telles normes, et les moyens techniques employés pour la réalisation d'objectifs consensuels. Cela, Majone le suppose vrai pour les règlements européens en matière de sécurité des produits, normes écologiques, et règles de concurrence, et pour le rôle de la banque centrale européenne dans une Union monétaire future. Mais ce ne serail pas vrai pour la politique sociale et tous les autres domaines politiques dans lesquels un conflit distributif (ou, par implication, conflit idéologique ou normatif) est endémique. Ici, l'autorité de l'expertise technique ne suffira pas ; de telles décisions dépendent plutôt d'une légitimité majoritaire - qui n'est pas disponible au niveau européen. En conséquence, les politiques concernant l'assistance sociale ne peuvent pas être des politiques européennes ; elles doivent ètre laissées aux Etats membres de l'Union où, probablement, les politiques redistributives peuvent être démocratiquement légitimées. Ce que Majone ne prend pas en compte, cependant, est le fait que la viabilité des Etats-providence nationaux est directement défiée par l'intégration économique européenne qui réduit radicalement l'efficacité de l'auto-détermination démocratique 
au niveau national. Cela constitue actuellement le problème central auquel est confronté tout gouvemement démocratique en Europe.

La force de l'intégration négative
Pour développer cet argument, je trouve utile de me référer à la distinction juridique entre "intégration négative" et "intégration positive". Tandis que la première se refère à l'abolition des obstacles nationaux à la libre circulation des biens, services, capitaux et personnes, l'intégration positive se réfère à l'européanisation des politiques publiques - ce qui est le sujet même du colloque. C'est la force de l'intégration négative qui est à la base des difficultés actuelles des Etats-providence européens, et c'est la faiblesse de l'intégration positive qui empêche la résolution de ces difficultés au niveau européen.

L'intégration négative était déjà implicite dans le texte original du Traité de Rome puisque les Etats contractant acceptaient l'obligation, non seulement d'éliminer toutes les barnères protectrices, mais encore toutes les barrières non-tarifaires qui pourraient empècher les interactions économiques et la libre concurrence parmi les Etats membres du "Marché Commun" européen. Ces dispositions du traité étaient interprétées par la Cour européenne de justice comme représentant non seulement de simples obligations internationales, mais comme devant prendre effet directement à l'inténeur des systèmes légaux des Etats membres, et devant s'imposer aux décisions administratives aussi bien qu'aux législations et aux constitutions nationales. Néanmoins, une fois que tarifs et restrictions quantitatives ont été abolis dans la première décennie du Marché Commun, l'élimination ultérieure des barrières nontanifaires dans les échanges fut assez lente dans la pratique. Ce ne fut qu'à partir du milieu des années 1980 que l'intégration négative allait connaître une nette accélération à travers le programme de marché unique de Jacques Delors et l'achèvement du marché inteme européen vers la fin de 1992.

En conséquence, et pour couper court à une longue histoire, nous avons maintenant, à l'intérieur de l'Union européenne, un domaine économique intégré, à l'intérieur duquel les restrictions légales et administratives contre la libre circulation des biens et des capitaux ont été complètement annulées, où les restrictions à la libre concurrence entre les fournisseurs de services ont été rapidement éliminées, et où les restrictions légales contre la mobilité des travailleurs et des étudiants sont à certains égards moins fortes qu'elles le sont même dans des Etats fédéraux confirmés comme les Etats-Unis d'Amérique1. De plus, ces quatre libertés sont vigoureusement renforcées contre toutes contraintes nationales rémanantes sur le commerce et toutes distorsions nationales à la libre concurrence, non seulement de la part de la Commission européenne et de la Cour européenne de Justice, mais aussi de la part des tribunaux

1 Par exemple, les universités d'Etat aux Etats-Unis imposent des frais de scolarité plus élevés aux étudiants des autres Etats, tandis que la Cour Européenne de Justice a décidé qu'aucun frais de scolarité ne pouvait être demandé aux étudiants d'un autre Etat-membre de l'Union Européenne, même si des frais sont â payer par les étudiants nationaux. 
nationaux dans les procès administratifs ou civils ordinaires. En pratique donc, les capitaux ont toute liberté de se déplacer là où les taux d'intérêt sont les plus élevés, et les entreprises sont libres de déplacer leurs activités de production et de recherchedéveloppement en tout lieu de l'Union sans compromettre en aucune façon leur accès à leur marché intérieur. En conséquence, cela a éliminé la capacité des gouvernements nationaux à protéger leurs entreprises nationales contre des concurrents produisant à l'étranger sous des régimes réglementaires différents, et leur capacité à imposer et réglementer les capitaux domestiques et les entreprises est maintenant limitée par la crainte de la fuite des capitaux et de la délocalisation de la production. De là, tous les gouvemements nationaux de l'Union européenne sont maintenant forcés d'entrer en concurrence avec chacun des autres dans le but d'attirer, ou de retenir, des entreprises et des capitaux mobiles. Dans ce sens, leur situation est maintenant comparable à celle des gouvemements subnationaux dans les marchés nationaux unifiés d'Etats fédéraux comme les Etats-Unis, le Canada, la République Fédérale d'Allemagne ou la Suisse.

Dans ces Etats fédéraux, les unités régionales de gouvemement - états, provinces, Länder ou cantons - ont longtemps été confrontées au fait que leur propre juridiction ternitoriale est plus étroitement circonscrite que les frontières du marché, et qu'elles sont constitutionnellement requises de respecter la liberté des mouvements de traversée des frontières et les transactions économiques. On doit s'attendre, donc, à ce que la répartititon traditionnelle des fonctions de gouvernement dans des Etats fédéraux, entre le centre et les régions, reflète le fait que les capitaux et les entreprises ont toujours été mobiles à l'intérieur des frontières du marché national. A partir de là, on pourrait tirer d'importantes leçons pour la répartition des fonctions de gouvernement dans l'Union européenne.

La solution la plus nette se trouve en République Fédérale d'Allemagne, où la concurrence réglementaire entre les Länder est presque totalement éliminée dans des domaines qui pourraient concemer les choix de localisation de capitaux et de firmes. Tous les impôts, y compris ceux revenant aux Länder, sont fixés par une législation fédérale uniforme ; il en est de mème de tous les impôts et bénéfices pour l'assistance sociale, y compris ceux qui sont payés en dehors des budgets des gouvemements locaux. Parallèlement, tous les règlements de sécurité et de santé, et tous les règlements écologiques qui pourraient concerner des entreprises sont uniformes à travers la nation, et il en est de même de la loi sur les bénéfices, la loi sur le travail, la loi sur les conventions collectives et, peu ou prou, les accords de convention collective négociés entre les syndicats nationaux et des associations d'employeurs. Ainsi, la fonction économique principale des gouvemements de Länder est la promotion des affaires en foumissant des infrastructures publiques, incluant l'éducation et la formation permanente, des facilités de recherche orientées vers l'industrie et les services d'institutions de transfert de technologie, tandis que des subsides financiers aux entreprises sont encore réglementés par la législation nationale.

Du point de vue international, le niveau de la centralisation régulatrice allemande est inhabituel. Néanmoins, tous les systèmes fédéraux ont été confrontés au même choix 
entre, d'un côté, reporter 'vers le haut', au niveau national, la responsabilité des politiques concernant les revenus du capital et les coûts de production des entreprises mobiles, ou éviter totalement de telles politiques, d'un autre côté1. En fait, les politiques sociales et l'assistance sociale, dans la mesure où elles existent, ou bien ont été 'nationalisées' ou bien, là où elles restent formellement au niveau régional, en sont arrivées à dépendre si lourdement des subsides nationaux que la responsabilité politique effective incombe aussi bien au gouvemement central.

Ainsi, la leçon à tirer de l'expérience d'Etats fédéraux semble aller dans la direction exactement contraire à celle suggérée par G. Majone : au niveau national, les politiques de l'Etat-providence qui auraient clairement une légitimité majoritaire sont de plus en plus contestées par la concurrence économique dans le manché européen intégré ; en conséquence, les gouvernements nationaux perdent de la légitimité démocratique dans la dimension orientée vers l'output ou la dimension de l'efficacité. A partir de là, si l'auto-détermination efficace doit être maintenue, la responsabilité des politiques redistributives devrait être transférée du niveau national au niveau européen. L'Europe, en d'autres termes, devrait suivre la voie prise par des Etats fédérés nationaux en développant la "dimension sociale" dans un Etat-providence européen global - ou, au moins, en harmonisant les politiques sociales nationales de façon à éliminer la concurrence ruineuse entre des régimes nationaux d'assurances sociales.

La faiblesse de l'intégration positive
Si l'on prenait cette voie, cependant, nous serions encore confrontés au déficit démocratique au niveau de l'Union européenne. Comme ce déficit ne pourrait pas être surmonté dans le proche avenir, des décisions ne pourraient pas être prises par un vote majoritaire au Parlement européen et par un gouvernement européen démocratiquement légitimé, mais continueraient à dépendre de l'accord des gouvemements nationaux au Conseil des Ministres et au Conseil européen. Beaucoup de ces décisions devraient encore être prises unanimement et, même en cas de vote à la majorité qualifiée, l'opposition d'un petit nombre de pays pourrait bloquer l'action européenne. Ainsi, l'Union européenne n'est elle capable d'une action efficace que dans des domaines dans lesquels les États membres ont des intérêts complémentaires ou convergents. De tels domaines existent, et ils coïncident essentiellement avec ces domaines de politique régulatrice évoquée par G. Majone. Mais la politique sociale et l'Etat-providence ne figurent pas parmi eux.

1 Un exemple instructif est foumi par l'histoire de la législation du travail des enfants aux Etats-Unis dans les premières décennies de ce siècle (Graebner 1977). Comme la réglementation des conditions de production était considérée comme outrepassant le pouvoir fédéral sur le commerce inter-Etats alors qu'il était interdit aux Etats d'interférer sur le commerce inter-Etats, les Etats qui essayaient de limiter l'emploi d'enfants dans la production industrielle trouvaient que leurs industries étaient menacées par des importations venant d'Etats où le travail des enfants était encore permis. Le travail des enfants a donc continué sans réduction, même dans les Etats "progressistes", jusqu'à ce que la "révolution constitutionnelle" du New Deal permette entin une réglementation au niveau fédéral après 1937. 
Cela s'explique par les différences extrêmes en matière de développement économique entre les Etats membres de I'Union. Dans sa composition actuelle, I'Union inclut certaines des économies nationales les plus développées dans le monde, et certaines économies qui sont à peine au-dessus du seuil de pauvreté. La productivité moyenne du travail, les salaires et les revenus par tête au Danemark sont environ six fois plus élevés qu'ils ne le sont au Portugal - une différence qui est beaucoup plus grande que celle existant entre les régions dans les Etats fédéraux arrivés à maturité. En conséquence, les Etats membres les moins développés économiquement ne peuvent simplement pas se permettre de surcharger leurs entreprises, travailleurs ou consommateurs au même niveau de coûts sociaux ou écologiques que les citoyens des Etats développés ont acceptés, voire exigés. Mais même si le conflit Nord-Sud à l'intérieur de l'Union pouvait être d'une certaine façon neutralisé, I'harmonisation des Etats-providence européens serait extrêmement difficile et probablement impossible.

En fait, la difficulté la plus importante n'est pas économique mais institutionnelle. Les Etats-providence européens se sont historiquement développés selon des formes très hétérogènes. Voyez la différence entre les systèmes sociaux universalistes des pays scandinaves d'une part, et les systèmes corporatistes de l'Europe continentale, d'autre part. Les premiers ont mis l'accent sur les services sociaux, les seconds sur le paiement de translerts sociaux. Les premiers ont des pensions de retraites basées sur l'impôt combinées avec des fonds de pension complémentaires fondés sur la contribution volontaire, tandis que les seconds ont des systèmes suivant le mode de répartition, c'est-à-dire que les pensions des inactifs sont financées par les actifs à travers des contributions obligatoires basées sur les salaires. Les pays scandinaves ont des services nationaux de santé tandis que les pays continentaux ont des systèmes de soins basés sur lassurance, dans lesquels les médecins privés sont payés sur la base d'honoraires. La liste pourrait être allongée, et devrait être étendue si le Royaume-Uni était comparé à l'un ou l'autre modèle, scandinave ou continental. Et les différences paraîtraient mêmes plus grandes si nous incluions les systèmes de relations industrielles dans notre définition des institutions d'Etats-providence .

Dans ces conditions, toute tentative d'harmonisation européenne requiert des changements institutionnels fondamentaux dans la plupart des systèmes nationaux existants, et nous devons nous attendre à des conflits féroces pour adopter au niveau européen le modèle institutionnel. Les pays qui seraient perdants dans cette bataille devraient alors réorganiser fondamentalement (ou même faire disparaître) les puissantes organisations qui font vivre des centaines de milliers, voire des millions d'employés et dont dépend une grande part de l'électorat qui bénéficie de leurs services et de transferts de revenus. Autrement dit, les difficultés politiques à harmoniser les structures institutionnelles d'Etats-providence arrivés à maturité seraient si accablantes que l'on comprend que personne, ni les gouvernements, ni les partis d'opposition, ni les associations d'employeurs ou les syndicats, n'exige actuellement que l'harmonisation de la politique sociale soit mise à l'agenda européen. 
Au lieu de cela, nous voyons que, dans tous les Etats membres, les gouvernements et les syndicats luttent contre les conséquences de la concurrence économique dans le marché interne. Partout, les syndicats acceptent des réductions de salaire, et les gouvernements déréglementent et abaissent les impöts sur les entreprises et sur les revenus des capitaux, tandis que les services publics, les bénéfices sociaux et les paiements de pension de retraites sont réduits. La Grande-Bretagne a pris la tête de ce mouvement ; la France a affronté ce défi au cours de l'hiver 1995 ; le gouvemement allemand a formulé en avril 1995 le programme d'austérité le plus sévère de l'après-guerre, et la Suède a suivi ce processus en juin de la même année. II peut encore y avoir des différences idéologiques entre les gouvernements qui utilisent la pression extérieure pour réduire le niveau de redistribution et de réglementation sociale, et les gouvemements qui rechignent face à cette contrainte économique. L'effet, cependant, est le mème : avec l'achèvement du marché interne européen, tous les Etats membres doivent entrer en concurrence entre eux pour attirer ou retenir des facteurs de production intemationaux mobiles - principalement des capitaux d'investissement, des entreprises, de la recherche et développement, et d'autres services hautement productifs -. En conséquence, tous se voient eux-mèmes obligés de réduire la charge de la réglementation et de l'impôt sur les capitaux. et les entreprises. Dans le mème temps, ils doivent accroitre les charges d'impôt sur les travailleurs et les consommateurs, et doivent résister et réduire les revendications de groupes - les jeunes, les malades, les chômeurs et les personnes âgées - qui dépendent des services publics et des transferts sociaux.

La fin de la démocratie?
Néanmoins, nous savons tous par des sondages d'opinion, des manifestations de masse et des résultats électoraux que l'Etat-providence continue à avoir un large soutien politique en Europe. Ainsi, les réductions actuelles du social ne peuvent pas être interprétées comme l'aboutissement de choix politiques démocratiquement légitimés. Au lieu de cela, chaque gouvernement les adopte et les défend sous la contrainte exteme de la nécessité économique. Cette nécessité, cependant, n'est pas survenue par accident; elle avait pour cause la décision politique d'achever le marché européen par l'Acte Unique européen de 1986 qui avait été ratifié, pratiquement sans opposition, par tous les parlements nationaux. Les difficultés qui ont suivi étaient assez faciles à prévoir, et avaient été correctement annoncées par beaucoup. Nous devons, donc, supposer que l'on pensait que ces difficultés seraient contrebalancées par les bénéfices économiques du marché interne (ou, inversement, par les prétendus "coüts de non-Europe"). Quoi qu'il en soit, le marché européen est intégré, ses conséquences économiques sont vraisemblablement irréversibles, et personne semble-t-il ne plaide sérieusement pour un retour à un régime de marchés nationaux protégés en Europe.

S'il en est ainsi, le dilemme qui a été causé par les choix politiques de gouvernements nationaux et de parlements démocratiquement responsables doit être affronté sans détour. Avec l'achèvement du marché interne, les gouvernements nationaux ne sont plus capables de poursuivre les politiques sociales et les politiques d'Etat-providence 
que leurs citoyens tiennent pour acquises et continuent à demander. Au niveau national, donc, l'auto-détermination démocratique a perdu de son efficacité. Dans le même temps, la tentative d'harmoniser les politiques sociales au niveau européen manquerait de légitimité démocratique authentique et, dans les structures institutionnelles existantes de l'Union, serait politiquement irréalisable.

Dans ces conditions, nous pourrions aussi bien déclarer la "fin de la démocratie" en Europe. Si nous ne voulons pas qu'il en soit ainsi, nous devons une fois de plus réexaminer notre concept de démocratie. Nous avons défini l'auto-détermination comme l'authenticité plus l'efficacité, signifiant ainsi que les gouvemements devraient être efficaces en réalisant les préférences non-manipulées des citoyens. Mais si nous devions appliquer cette définition à l'auto-détermination individuelle, cela décrirait les attentes d'une personne passablement immature. Ce qui manque est ce que Freud appelait le "principe de réalité" par lequel des fantaisies infantiles d'omnipotence sont tempérées par une évaluation réaliste de ses propres limitations et possibilités. Dans le mème sens, les démocraties arrivées à maturité ne peuvent pas mettre sur le même plan l'auto-détermination avec l'omnipotence politique. Les gouvernements démocratiques doivent prendre la responsabilité des conséquences économiques de leurs choix politiques, et les publics démocratiques doivent définir leurs préférences à la lumière de ce que sont des aspirations réalisables sous les contraintes externes prévalantes.

Ceci ne signifie pas qu'avec l'achèvement du marché inteme européen tous les buts et aspirations doivent être abandonnés, qui avaient dans le passé été associés aux Etats-providence au niveau national. Mais ils doivent certainement ètre re-spécifiés à la lumière de ce que les Etats-nations sont encore capables de réaliser. Parallèlement, nous ne devons pas simplement renoncer aux espoirs qui avaient été associés à l'intégration positive au niveau européen. Mais nous devons les re-spécifier dans les termes de ce que l'Union européenne, dans sa forme institutionnelle actuelle, est capable ou incapable de réaliser. Dans le contexte actuel, il est difficile d'énumérer toutes les implications concrètes de cette invocation du principe de réalité. Mais quelques exemples sont nécessaires pour illustrer sa potentialité.

Commençons par quelques options stratégiques qui existent encore au niveau national:

- Si les termes de l'échange entre le capital et le travail ont de façon irréversible évolué en faveur du capital, les partis politiques et les syndicats qui souhaitent encore poursuivre des buts égalitaires devraient transférer leur attention de la politique des salaires à la distribution des actions de capitaux.

Si la tentative d'entretenir la concurrence intemationale en diminuant les coùts des entreprises détruit l'Etat-providence, les gouvemements et les syndicats doivent se concentrer sur la création pour les entreprises des avantages locaux accroissant leur productivité. 
- Si le financement des dépenses sociales par des impôts sur les entreprises et par des coûts sur le travail non-salarié conduit à la fuite des capitaux, au désinvestissement et à la perte d'emplois, une partie croissante du fardeau social doit être transférée aux impôts sur la consommation.

- Si les pertes d'emplois dans les secteurs intemationalement exposés de l'économie nationale ne peuvent pas être empêchées, les gouvemements doivent prolonger leurs efforts de politique industrielle par l'amélioration des conditions de la croissance de l'emploi dans les secteurs servant la demande locale des individus et des ménages.

- Si l'Etat-providence est surchargé par l'accroissement du chômage, les gouvemements doivent essayer d'accroitre le "contenu en emplois' des dépenses sociales - par exemple en subventionnant les emplois à bas-salaire au lieu de financer le chômage à plein-temps.

Parallèlement, au niveau européen, on pourrait illustrer, par les suggestions suivantes, que les Etats membres pourraient en réalité accepter une concentration sur les stratégies pouvant réduire la concurrence.

- Si les conflits d'intérêt surviennent principalement des différences de niveau de développement économique entre les Etats membres, un accord sur I'harmonisation pourrait quand même être atteint sur des règlements stipulant des normes dédoublées - l'une définie au niveau d'aspiration des pays plus avancés, et l'autre définie au niveau que peuvent s'offrir les Etats membres économiquement moins développés.

- Si les conflits d'intérêt surviennent principalement à cause des différences institutionnelles parmi les Etats membres, un accord pourrait quand même être atteint sur l'harmonisation des niveaux quantitatifs d'effort - comme des parts minimum du PIB qu'un pays confierait aux services de l'Etat-providence et aux transferts, indépendamment de la forme dans laquelle ceux-ci sont fournis ou financés.

- Tout comme le Traité contient des interdictions strictes de subsides nationaux qui pourraient modifier la concurrence dans le marché interne, l'Union pourrait adopter et appliquer des interdictions similaires contre des déréglementations concurrentielles.

- Les pays qui ont pris conscience des conséquences déflationnistes des tentatives nationales pour satisfaire les critères de Maastricht pourraient être capables de s'entendre sur une coordination de leurs stratégies macro-économiques à l'échelle européenne. 
- Les pays qui sont devenus conscients des dommages entrainés par la compétition en matière d'impôts et taxes pourraient être capables de s'entendre sur l'harmonisation des impôts nationaux sur les revenus des capitaux et les profits des fimes.

Ces listes ne sont certainement ni exhaustives, ni définitives. Mais elles suggèrent que, même sous les conditions d'un marché inteme pleinement intégré en Europe, des opportunités existent encore au niveau national pour des choix politiques efficaces et significatifs. Il est vrai qu'elles ne permettent pas la poursuite de solutions qui étaient fructueuses et ont trouvé un soutien démocratique dans les décennies d'aprèsguerre. Elles requièrent des changements de stratégie particulièrement douloureux pour les syndicats de travailleurs et les partis de gauche. Mais elles n'impliquent pas la capitulation des politiques démocratiques devant la force écrasante des marchés capitalistes.

Dans le même temps, l'Union européenne ne peut pas être transformée dans le proche avenir en une entité politique démocratique, et elle ne peut pas non plus assumer les fonctions de l'Etat-providence national. Mais les institutions de l'Union européenne peuvent être utilisées pour permettre et conforter des solutions politiques nationales qui défendront les droits de citoyenneté et les valeurs de l'Etat-providence contre les forces du marché intégré. Quand ces opportunités seront saisies, l'avenir de la démocratie en Europe semblera loin d'être aussi sombre que ce que le suggère souvent le débat actuel. 